\title{
Th17 cells in systemic lupus erythematosus share functional features with Th17 cells from normal bone marrow and peripheral tissues
}

\author{
Ana Henriques • Luís Inês • Maria Luísa Pais • \\ José António Pereira da Silva • Artur Augusto Paiva
}

Received: 18 March 2011 /Revised: 23 August 2011 /Accepted: 18 September 2011

(C) Clinical Rheumatology 2011

\begin{abstract}
This study was designed to investigate the functional heterogeneity of human Th17 and how their plasticity shapes the nature of immune cell responses to inflammation and autoimmune diseases, such as systemic lupus erythematosus (SLE). We evaluated functional Th17 cell subsets based on the profile of cytokine production in peripheral blood (PB), bone marrow aspirates (BM) and lymph node biopsies (LN) from healthy individuals $(n=35)$ and PB from SLE patients $(n=34)$. Data were analysed by an automated method for merging and calculation of flow cytometric data, allowing us to identify eight Th17 subpopulations. Normal BM presented lower frequencies of Th17 ( $p=0.006$ and $p=0.05)$ and lower amount of IL-17 per cell ( $p=0.03$ and $p=0.02)$, compared to normal $\mathrm{PB}$ and $\mathrm{LN}$ biopsies. In the latter tissues were found increased proportions of Th17 producing TNF- $\alpha$ or TNF- $\alpha / \mathrm{IL}-2$ or IFN- $\gamma / \mathrm{TNF}-\alpha / \mathrm{IL}-2$, while in BM, Th17 producing other cytokines than IL-17 was clearly decreased. In SLE patients, the frequency of Th17 was higher than in control, but the levels of IL-17 per cell were significantly reduced $(p<0.05)$. Among the eight generated subpopulations, despite the great
\end{abstract}

Ana Henriques and Luís Inês contributed equally to this work.

A. Henriques $\cdot$ M. L. Pais $\cdot$ A. A. Paiva $(\bowtie)$

Cytometry Service of the Histocompatibility Center of Coimbra,

Portugal, Histocompatibility Center of Coimbra,

Edifício São Jerónimo, $4^{\circ}$ Piso, Praceta Mota Pinto,

3001-301 Coimbra, Portugal

e-mail: apaiva@histocentro.min-saude.pt

L. Inês · J. A. P. da Silva

Rheumatology Service of the University Hospitals of Coimbra, Portugal,

Avenida Bissaya Barreto,

3000-075 Coimbra, Portugal

L. Inês

Rheumatology Service of the University Hospitals of Coimbra, Faculdade de Ciências da Saúde, Universidade da Beira Interior, Covilhã, Portugal functional heterogeneity of Th17 in SLE, a significant low proportion of Th17 producing TNF- $\alpha$ was found in inactive SLE, while active SLE showed a high proportion producing only IL-17. Our findings support the idea that the functional heterogeneity of Th17 cells could depend on the cytokine microenvironment, which is distinct in normal $\mathrm{BM}$ as well as in active SLE, probably due to a Th1/Th2 imbalance previously reported by our group.

Keywords Bone marrow - Lymph node biopsies Pro-inflammatory cytokines · Systemic lupus erythematosus . Th17

\section{Introduction}

Although it has been known for over 10 years that activated CD4 + T cells produce IL-17, the existence of a third subset of CD4+ effector $\mathrm{T}$ helper cells, named Th17 cells, has only recently been identified as an independent $T$ cell lineage, distinct from classical Th1 and Th2 cells [1-3]. Nevertheless, a common developmental origin between human Th17 and Th1 cells has been described in various studies [4-6]. Accordingly, human Th17 clones appear to express IL$12 \mathrm{R} \beta 2$ in addition to IL-23R, and the transcription factor Tbet in addition to ROR $\gamma \mathrm{t}$, as well a remarkable proportion of human Th17 cells, produces both IL-17A and IFN- $\gamma$, or human Th17 clones can be induced to produce IFN- $\gamma$ and upregulate T-bet expression when cultured in the presence of IL12 [7]. This close relationship between Th1 and Th17 cells is also marked in their capacity to cause T-cell-mediated inflammation and autoimmune disease. In fact, Th17 cells have been found to have a pathogenic role in several autoimmune disorders, including experimental autoimmune encephalomyelitis [8], rheumatoid arthritis $[9,10]$, inflammatory bowel disease [11], systemic sclerosis [12] and systemic lupus erythematosus 
(SLE) [13, 14], which were previously thought to be mainly caused by Th1 responses. Interestingly, new studies show Th17 cells as more potent than Th1 cells in inducing disease [15], linking the cytokine IL-17 in coordinating tissue inflammation via the up-regulation of additional proinflammatory and neutrophil-recruiting cytokines and chemokines, suggesting a pro-inflammatory role in these conditions. Moreover, in Crohn's disease, the existence of a remarkable number of double positive IFN $\gamma+/$ IL17 + T cells, named Th17/ Th1 cells, as well as the possibility to generate Th17/Th1 clones was reported [16]. These latter observations further support the concept that both Th1 and Th17 cells could contribute to the maintenance of inflammation in such disorders via the production of IFN $\gamma$ and IL-17. However, whether human autoimmune disorders, including SLE, are prevalently Th1-mediated or Th17-mediated and how they might cooperate with one another to perpetuate the inflammatory response are still unclear.

In the present study, we determined the relative frequency of Th17 cells (through their expression of IL17) in peripheral blood samples (PB), bone marrow aspirates (BM) and lymph node biopsies (LN), simultaneously quantifying the frequency of these cells producing IL-2, TNF- $\alpha$ and IFN- $\gamma$. Using an automated method of integration of flow cytometric data from several multicolour stains of the same cell sample into a single multicolour staining data file [17], we described the functional heterogeneity of human Th17 cells, identifying eight different Th17 cell subpopulations.

Considering the well-defined role played by cytokines in the immune deregulation observed in autoimmune diseases, we compared the distribution of the eight identified Th17 subpopulations in a group of SLE patients and age-matched healthy control subjects, suggesting that their particular functional behaviour may differ according to the microenvironment condition and participate dynamically in autoimmune response.

Taken together, our data shed new light on the nature of human Th17 cells and their close association with Th1 cells, strengthening the relevance of knowing how their great functional plasticity may influence the disease activity of SLE.

\section{Methods}

Control samples

We recruited 10 bone marrow aspirate samples $(70 \%$ female, mean age \pm SD: $58.3 \pm 20.1$ years), 15 peripheral blood samples $(73 \%$ female, mean age \pm SD: $33.8 \pm$ 9.8 years) and 10 lymph node biopsies (50\% female, mean age \pm SD: $48 \pm 16.1$ years) from healthy individuals at the
Histocompatibility Center of Coimbra; all were free from autoimmune disease and active infection and were not undergoing treatment with immunomodulatory drugs for any known condition. Pregnancy and age below 18 were exclusion criteria for participation. Bone marrow aspirate and peripheral blood samples were collected from healthy donors, while lymph node biopsies were collected from healthy donor victims of traffic accidents, and organs were received at the Histocompatibility Center of Coimbra to perform histocompatibility testing.

Samples from both controls and patients were sent to the laboratory, identified with a code number, blinding the laboratory to the disease status. The study protocol was approved by a local ethics committee, and all participants gave their signed informed consent.

\section{Patients}

We recruited patients fulfilling the 1997 American College of Rheumatology classification criteria for SLE [18] followed at the Rheumatology Service of the University Hospitals of Coimbra, Portugal. After assessing disease activity at the time of evaluation according to the SLE Disease Activity Index (SLEDAI, 2000) [19, 20], the PB samples from the 34 SLE patients were divided in two groups, one with active (SLEDAI $\geq 5 ; n=15$ comprising $86.7 \%$ female, mean age $32 \pm 11$ years) and the other with inactive (SLEDAI $<5 ; n=19$ comprising $89.5 \%$ female, mean age $34 \pm 9$ years) SLE [21]. The patient's active medication at the time of evaluation was recorded. The clinical features of SLE patients are presented in Table 1.

Table 1 Clinical findings in 34 patients with systemic lupus erythematosus (SLE)

\begin{tabular}{lll}
\hline & $\begin{array}{l}\text { Active SLE } \\
(n=15)\end{array}$ & $\begin{array}{l}\text { Inactive SLE } \\
(n=19)\end{array}$ \\
\hline Mean SLEDAI scores & $10.3 \pm 3.53$ & $2.7 \pm 1.52$ \\
Mean time since diagnosis & $9 \pm 5$ & $9 \pm 5$ \\
Lupus nephritis & $66.7 \%$ & $52.6 \%$ \\
Neurolupus & $\mathrm{NF}$ & $20 \%$ \\
Lupus arthritis & $93.3 \%$ & $68.4 \%$ \\
Haematological involvement & $93.3 \%$ & $84.2 \%$ \\
Lupus skin disease & $80 \%$ & $52.6 \%$ \\
Treatment & & \\
Hydroxychloroquine & $\mathrm{a}$ & $94.7 \%$ \\
Prednisone $^{\mathrm{a}}$ & $80 \%$ & $42.1 \%$ \\
Immunossupressants $^{\mathrm{b}}$ & $93.3 \%$ & $31.6 \%$ \\
\hline
\end{tabular}

$N F$ not found, $n$ sample investigated

${ }^{\mathrm{a}}$ Mean daily dose 18.2 and $5.3 \mathrm{mg}$

${ }^{\mathrm{b}}$ Micofenolate mofetil, azatioprine or cyclosporine 
Immunofluorescent staining of peripheral Th17 subsets after in vitro stimulation with PMA/ionomycin in the presence of Brefeldin A

We diluted $500 \mu \mathrm{L}$ of each sample L/L (vol/vol) in RPMI1640 medium (Gibco; Paisley, Scotland, UK), supplemented with 2-mML-glutamine. We added $50 \mathrm{ng} / \mathrm{mL}$ of phorbol 12 myristate 13-acetate (PMA; Sigma, Saint Louis, MO, USA), $1 \mu \mathrm{g} / \mathrm{mL}$ of ionomycin (Boehringer Mannheim, Germany) and $10 \mu \mathrm{g} / \mathrm{mL}$ of Brefeldin A (Golgi plug-Sigma, Saint Louis, MO, USA) and incubated for $4 \mathrm{~h}$ at $37^{\circ} \mathrm{C}$ in a humidified incubator with $5 \% \mathrm{CO}_{2}$ concentration.

Each cultured sample was aliquoted and stained in three different tubes $(200 \mu \mathrm{L} /$ tube $)$ using an intracytoplasmatic permeabilization and staining protocol in order to separately analyse the intracellular expression of IL-2, TNF- $\alpha$ and IFN- $\gamma$ in IL-17-positive T cell subpopulations, within the CD4+ T cell subset. All cell aliquots were stained with anti-IL-17 PE (clone 41802; R\&D Systems, Europe) and separately with anti-IL-2 FITC (clone MQ1-17H12; BD Pharmingen, San Diego, CA, USA), anti-TNF- $\alpha$ FITC (clone MAb11; BD Pharmingen, San Diego, C.A., USA) and anti-IFN- $\gamma$ FITC (clone 4S.B3; BD Pharmingen, San Diego, CA, USA) according to the manufacturer's instructions for fixation and permeabilization. These monoclonal antibodies (MAbs) were added to each tube after staining cells for surface expression of MAbs directed against $\mathrm{T}$ lymphocytes subsets - anti-CD3PerCP (clone SK7; BD, San Jose, CA, USA) and anti-CD8 APC (clone SK1; BD, San Jose, CA, USA). Among positive $\mathrm{CD} 3$ cells, CD4+ $\mathrm{T}$ cells were identified after exclusion of the $\gamma \delta$-T cell subset according to their higher reactivity with anti-CD3 monoclonal antibody and typical light scatter. The election of CD8 instead of CD4 was made concerning the down-regulation of the CD4 co-receptor after in vitro stimulation, which is a fact that did not potentially influence the merging/calculating procedure since, in our study, five parameters were measured in common - forward light scatter (FSC), side light scatter (SSC), IL-17 PE, CD3 PerCP and CD8 APC - given a great security in the calculation of all the other parameters (IL-2 FITC, TNF- $\alpha$ FITC and IFN- $\gamma$ FITC) in three separated four-colour staining of the same cell sample.

Flow cytometry data acquisition and analysis

Data acquisition was performed in two consecutive steps in FACSCalibur flow cytometer (BDB, San Jose, CA) equipped with an argon ion laser and a red diode laser. In the first step, $2 \times 10^{4}$ events/test, corresponding to all nucleated cells present in the sample, were collected. To improve the sensitivity of the analysis of $\mathrm{T}$ cell subsets present at low frequencies in the different tissue samples, in the second step, information on $\mathrm{CD} 3+$ cells and typical light scatter contained on a minimum of $3 \times 10^{5}$ events from the total sample cellularity was specifically stored through an electronic live gate. To identify the different $\mathrm{T}$ cell subsets and evaluate cytokine production, we used the Infinicyt $^{\mathrm{T}}{ }^{\mathrm{T}}$ software program (Cytognos, Spain). Results illustrate the percentage of positive cells within each cell subset or/and their mean fluorescence intensity (MFI).

Merging of flow cytometry data files and estimation of simultaneous cytokine production in a single file

As shown in Fig. 1, cytokine production was measured in a single multicolour data file obtained after merging the original four-colour-staining (six-parameter) data files from each sample using the Infinicyt ${ }^{\mathrm{TM}}$ software program (Cytognos, Spain) [17]. The basic concepts behind such strategies require (1) merging of different data files corresponding to distinct aliquots of a sample, (2) virtual correction of differences between corresponding cell populations present in different aliquots of the same sample and (3) calculation of immunophenotypic data measured in one sample aliquot to the corresponding/similar cells measured in the other aliquots of the same sample. In this sense, it required the inclusion of backbone reagents, such as antiIL-17, -CD8 and -CD3, aimed at the identification of the cell population of interest-Th17 cells-in all MAb combinations to increase the reproducibility of the gating strategy used to select specifically these cell populations in a sample for the evaluation of their overall cytokine production (IL-2, TNF- $\alpha$, INF- $\gamma$ and IL-17). This strategy required strict supervision by an experienced operator since automated adjustment of gates between different data files may result in inappropriate detection of specific cell populations.

Sequential merging of all data files from several multicolour staining of the same cell sample was performed, and the calculation function of the Infinicyt ${ }^{\mathrm{TM}}$ software was based on nearest-neighbour statistical tools. Accordingly, in our study, five parameters were measured in commonforward light scatter (FSC), side light scatter (SSC), IL-17 PE, CD3 PerCP and CD8 APC - in three separated fourcolour stainings of the same cell sample; all other parameters (IL-2 FITC, TNF- $\alpha$ FITC and IFN- $\gamma$ FITC) were measured only for that subset of cellular events corresponding to the specific three-colour staining.

Briefly, for each event, a vector in a three-dimensional space was built-up based on the data measured for the five common parameters (FSC, SSC, IL-17 PE, CD3 PerCP and CD8 APC). Then, the nearest neighbour for each individual event in a data file/sample aliquot was calculated as that event in another file/aliquot showing the shortest distance to it in the three-dimensional space generated by those parameters measured in common in 

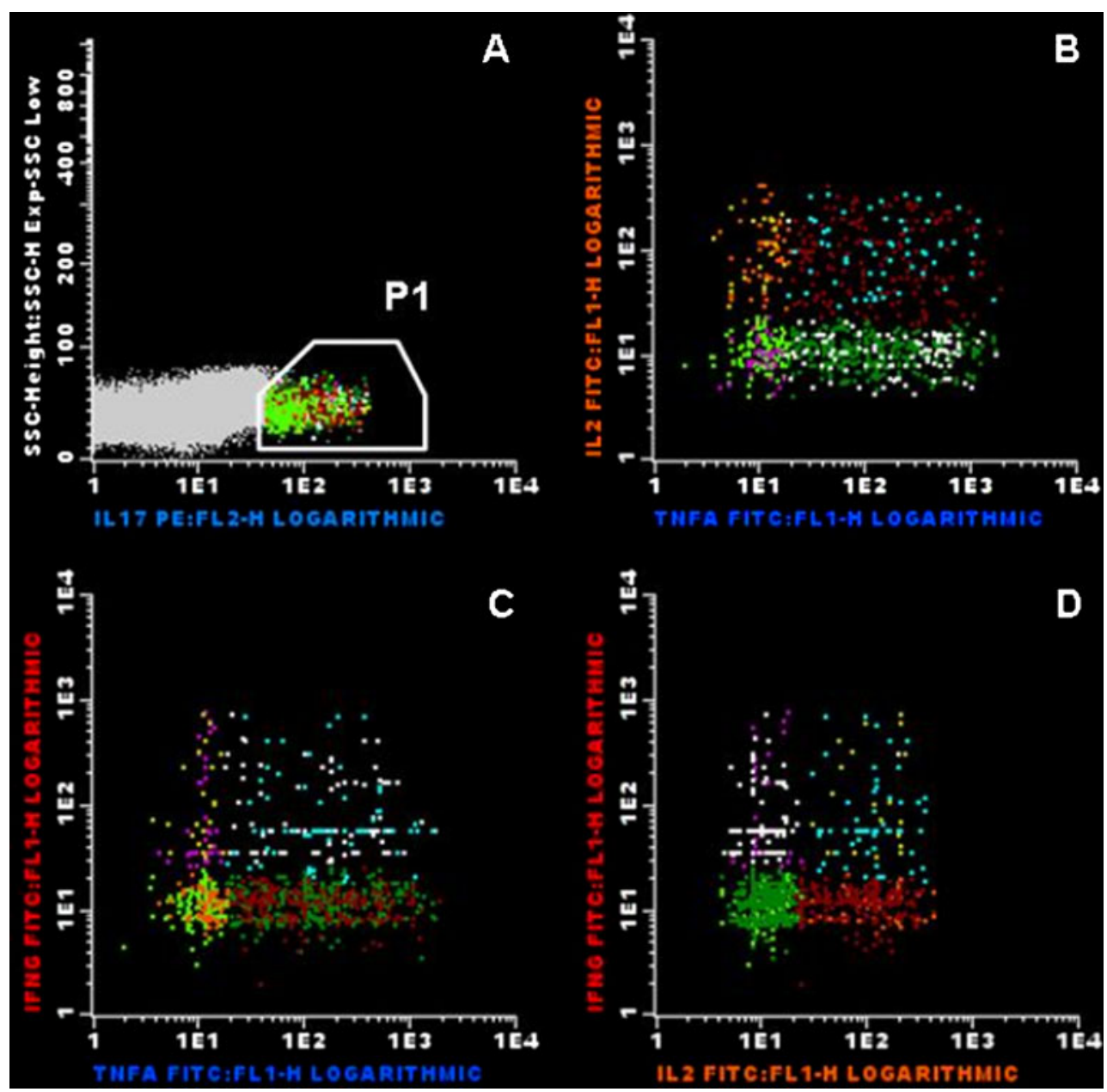

Fig. 1 Bivariate dot plot histograms illustrating the intracellular expression of IL-17, IL-2, TNF- $\alpha$ and IFN- $\gamma$ in Th17 cells after the merging and calculation processes performed on a set of three original four-color-staining data files corresponding to three aliquots of a representative $\mathrm{PB}$ sample from a ASLE patient, according to five common parameters - forward light scatter (FSC), side light scatter (SSC), IL-17 PE, CD3 PerCP and CD8 APC - and stained separately with IL-2, TNF- $\alpha$ and IFN- $\gamma$. Firstly, a gate was set in the whole merged/calculated data file (e.g., for both actually measured and

both data files/sample aliquots. Then, for each individual event in a data file, those values obtained for each of the closest events in the other data files were assigned for each of those parameters not actually measured in the former event.

\section{Statistical analysis}

Data were analysed using the non-parametric MannWhitney U test. Results were expressed as mean \pm SD and median. All statistical analyses were performed using SPSS (SPSS Inc., Chicago, USA), and differences were considered statistically significant when the $p$ value was less than 0.05 . calculated events) to define $\mathrm{T}$ cells; this gate (CD3 gate) was set in FSC versus SSC and SSC versus CD3 bivariate dot plot histograms. In addition, two-dimensional dot-plot representations, corresponding to combinations of antibodies (IL2, TNF- $\alpha$, INF- $\gamma$ ) conjugated with the same fluorochrome (FITC) but not obtained by direct staining of cells, were generated prior a "P1 gate" to the identification of "calculated Th17 cells" (a). Among the latter, eight "calculated Th17 subpopulations" were identified combining the three FITC-conjugated Mab (b-d)

\section{Results}

Frequency of human Th17 cells in different samples from healthy individuals

As shown in Table 2, the frequency of Th17 among CD4 T cells in the BM from healthy individuals was significantly lower than that found in control PB $(p=0.006)$ and LN samples $(p=0.02)$. The amount of IL-17 produced at single cell level (mean fluorescent intensity-MFI) was also markedly decreased in the BM, when compared to PB ( $p=$ $0.03)$ and $\mathrm{LN}(p=0.02)$ samples, with the amount of IL-17 being significantly higher in LN $(p=0.003)$ than in $\mathrm{PB}$ (Table 2). 
Table 2 Frequency of human Th17 in normal tissues and in PB from SLE patients

\begin{tabular}{lccccc}
\hline Th17 & BM & PB & LN & ASLE & ISLE \\
\hline $\begin{array}{l}\text { Percent of Th17 cells from } \\
\text { total CD4 }{ }^{+} \text {T cells }\end{array}$ & $0.6 \pm 0.2(0.5)^{*}$ & $1.6 \pm 0.7(1.4)$ & $1.6 \pm 0.7(1.5)$ & $1.9 \pm 0.9(1.9)$ & $2.0 \pm 1.2(1.7)$ \\
MFI & $63.9 \pm 25.5(61.2)^{* *}$ & $90.0 \pm 20.2(85.0)^{\#}$ & $157.4 \pm 50.9(179.0)$ & $65.8 \pm 16.7(63.9)^{ף}$ & $62.8 \pm 20.7(60.3)^{\S}$ \\
\hline
\end{tabular}

Results are expressed as mean \pm standard deviation (median). Statistically significant differences were considered when $p<0.05$ (MannWhitney U test): ${ }^{*} p=0.006,{ }^{* *} p=0.03 \mathrm{BM}$ versus $\mathrm{PB} ;{ }^{*} p=0.05,{ }^{* *} p=0.02 \mathrm{BM}$ versus $\mathrm{LN} ;{ }^{*} p=0.003$ PB versus LN; ${ }^{\Uparrow} p=0.006 \mathrm{ASLE}$ versus $\mathrm{PB} ;{ }^{\S} p=0.003$ ISLE versus PB

$M F I$ mean fluorescence intensity of positive cells, $P B$ peripheral blood, $B M$ bone marrow, $L N$ lymph node biopsies, $A S L E$ active disease, ISLE inactive disease

Functional characterization of human Th17 cells in normal tissues using an automated flow cytometric method

To further our investigation of the functional activity of Th17 cells in different tissues in healthy individuals, we studied the percentage of cells producing different combinations of pro-inflammatory cytokines.

As shown in Fig. 1, we identified eight distinct Th17 cell subpopulations in healthy subjects, according to their pattern of cytokine expression (TNF- $\alpha$, IL-2 and INF- $\gamma$ ), using an automated flow cytometric method for merging and calculation of data files.

We found that the majority of normal BM Th17 cells did not produce type 1 cytokines, resulting in a large subpopulation of cells showing isolated IL-17 expression (Fig. 2). In contrast, in both PB and LN samples, clearly distinct subpopulations of Th17 cells producing different cytokine combinations were identified with a consequent decrease in the frequency of the subpopulation producing isolated IL-17 ( $p=0.02$ and $p=$ 0.003 , respectively), a difference which was particularly marked in LN Th17 cells (Fig. 2). The proportion of Th17 cell subpopulation simultaneously producing all studied cytokines was significantly higher in both peripheral tissues (PB: $p=0.02$; LN: $p=0.006$ ), especially in LN compared to BM. The Th17 cell subpopulation simultaneously producing TNF- $\alpha$ and IL-2 was significantly more represented in LN than in PB or BM (LN versus BM: $p=0.003$; LN versus PB: $p=0.005$; PB versus BM: $p=0.02$ ). Those Th17 cells producing only TNF- $\alpha$ were also significantly increased in PB (PB versus BM: $p=0.003$; PB versus $\mathrm{LN}: p=0.002$ ) but markedly decreased in LN when compared to BM $(p=0.04)$, and Th17 cells producing only IFN- $\gamma$ were poorly represented in both peripheral tissue comparatively to BM (PB: $p=$ 0.02 and LN: $p=0.003$ ) (Fig. 2).

Frequency of human Th17 cells in peripheral blood in SLE patients

We found a trend for a higher frequency of Th17 among CD4 $\mathrm{T}$ cells in both active and inactive SLE, but did not

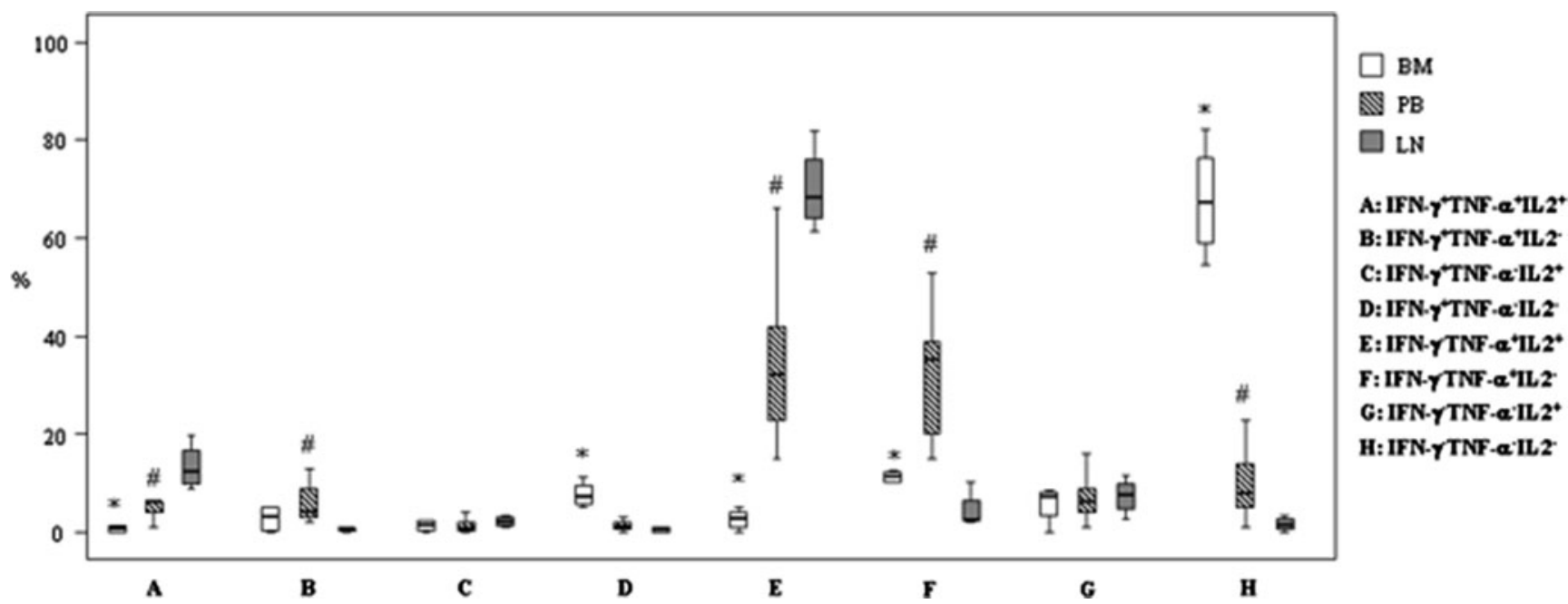

Fig. 2 Frequency of multiple subsets of human Th17 cells in BM, PB and $\mathrm{LN}$ from healthy subjects, according to the production of IL-2, IL$17, \mathrm{TNF}-\alpha$ and IFN- $\gamma$, after in vitro stimulation with PMA/ionomycin in the presence of Brefeldin A. Data were analysed by an automated flow cytometric method based in merging file calculation, using Infinicyt software. $B M$ bone marrow aspirates, $P B$ peripheral blood, $L N$ lymph node biopsies. Statistically significant differences $(p<0.05)$ when compared: *BM versus $\mathrm{PB}$ and $\mathrm{APB}$ versus $\mathrm{LN}$ 
reach statistical significance (Table 2). No differences were observed in the frequency of Th17 cells between active SLE and inactive SLE groups. However, analysis of the amount of IL-17 produced at single cell level (MFI) showed a significant decreased expression of IL-17 in Th17 cells of both SLE groups compared to normal controls (ASLE: $p=$ 0.006; ISLE: $p=0.003$ ) (Table 2).

Functional characterization of human PB Th17 cells in SLE patients using an automated flow cytometric method

Since current evidence suggests that there is an increased expression of IL-17 in patients with a variety of autoimmune diseases and a possible developmental relationship between the Th17 and Th1 phenotypes, we compared the pattern of distribution of the eight different proinflammatory cytokine-producing Th17 cell subpopulations in PB samples from SLE and normal controls. Despite the Th17 cell heterogeneity observed in PB from SLE patients, the pattern of distribution of the different cytokineproducing Th17 cell subpopulations observed was relatively similar to normal subjects (Fig. 3); however, a significant decrease in the Th17 cell subpopulations producing only TNF- $\alpha$ and IFN- $\gamma$ plus IL-2 was found in inactive SLE patients compared to normal controls $(p=0.03)$ and to active SLE groups $(p=0.02)$, respectively (Fig. 3). Interestingly, we observed a higher proportion of Th17 cells producing IL-17 alone in active SLE patients comparatively to the other studied groups but as it occurs for other subpopulations, without significant differences between groups (Fig. 3).

\section{Discussion}

In this study, we have applied a novel software program to map out the functional phenotype of Th17 cells according to their pro-inflammatory cytokine production profile, either in normal tissues or peripheral blood from SLE patient, to clarify the relevance of those different microenvironment conditions in Th17-cell differentiation.

Data published in the past few years suggest the existence of a novel subset of Th effectors that are distinct from the classic Th1 and Th2 and that have been named Th17 because of their ability to produce IL-17. Identified by IL-17 production, we showed in Table 2 that a clear decreased proportion of the Th17 cell population was observed in normal $\mathrm{BM}$ as compared to both normal peripheral tissues (PB and $\mathrm{LN}$ ), with no significant differences between the latter groups. When we analysed the amount of IL-17 expressed at the single-cell level, we observed a significant increase in both normal peripheral tissues, particularly in LN (Table 2).

As measured by other studies [16, 22, 23], a remarkable proportion of Th17 cells also shares the ability to produce IFN- $\gamma$. These cells, producing both IL-17 and IFN- $\gamma$, are usually named Th17/Th1. This new subset of IFN- $\gamma$ producing Th17 cells sharing features with both Th1 and Th17 challenged us to evaluate other pro-inflammatory cytokines that could raise new issues on the Th17 developmental and/or functional relationship with Th1 or even clarify its role in autoimmune disorders, such SLE. In this sense, the use of an automated flow cytometric method allowed us to identify eight different merged/calculated

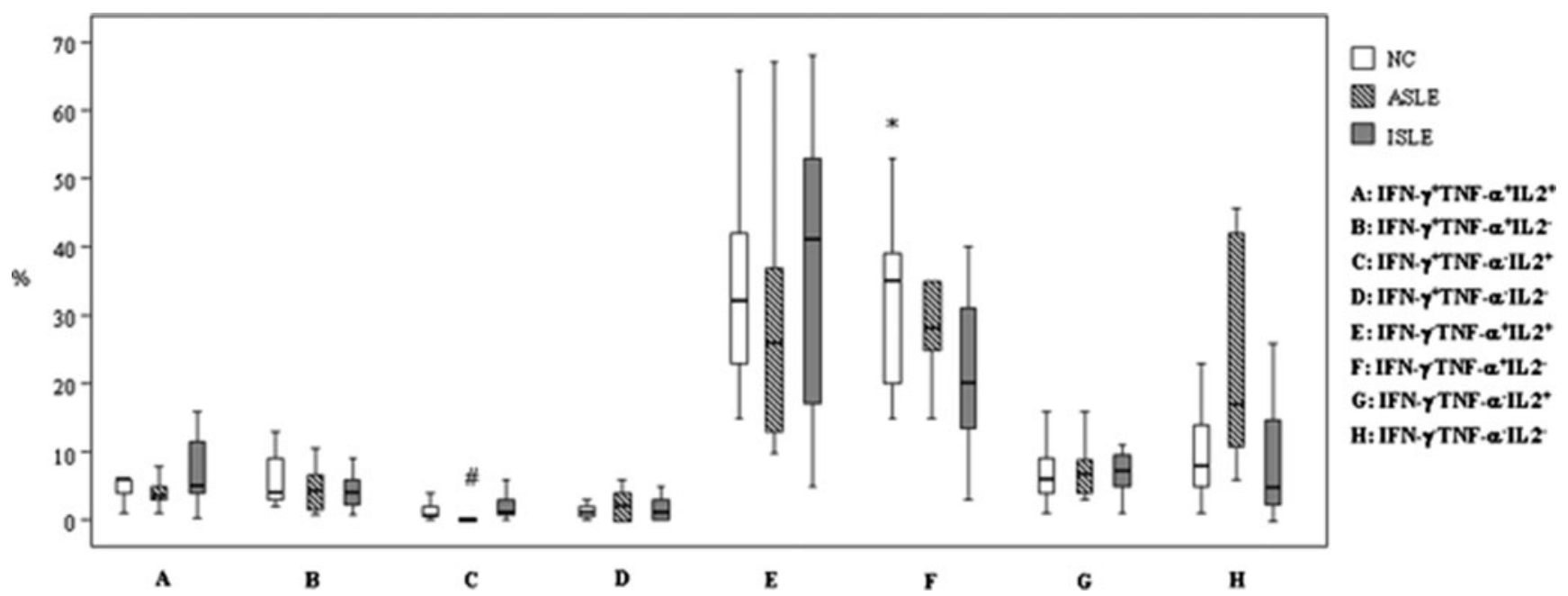

Fig. 3 Frequency of multiple subsets of human PB Th17 cells from NC, ASLE and ISLE patients, according to the production of IL-2, IL-17, TNF- $\alpha$ and IFN- $\gamma$, after in vitro stimulation with PMA/ionomycin in the presence of Brefeldin A. Data were analysed by an automated flow cytometric method based in merging file calculation, using Infinicyt software. $P B$ peripheral blood, $N C$ healthy individuals, $A S L E$ active disease, ISLE inactive disease. Statistically significant differences $(p<0.05)$ when compared: *NC versus ISLE; \# ISLE versus ASLE 
Th17 subpopulations from the functional point of view. We also demonstrated that Th17 cells in peripheral tissues (PB and LN) produce not only IFN- $\gamma$ but also TNF- $\alpha$ and/or IL2 (Fig. 2). BM Th17 cells showed a distinct profile of cytokine production, with the majority not secreting the type 1 cytokines studied. Moreover, a decreased amount of each cytokine was observed in normal BM as compared to $\mathrm{PB}$ and LN, without significant differences between those peripheral tissues (data not showed). Although predominately found in the lung and digestive mucosa, we demonstrated that Th17 cells can be observed in BM, PB and lymphoid tissues throughout the body. However, if Th17 cells represent terminally differentiated cells or whether they retain plasticity and can develop into another lineage, such as IL-2, TNF- $\alpha$ and/ or IFN $\gamma$ secreting Th1 cells, remains unproved.

In our knowledge, this is the first study that compares the relative distribution and functional activity of Th17 cells in normal $\mathrm{BM}, \mathrm{PB}$ and $\mathrm{LN}$ according to cytokine production, and it is also the first application of an automated flow cytometric method capable of defining eight different Th17 subpopulations, based on distinct cytokine expression combinations, after in vitro stimulation. The demonstration of the reliability of this new statistical approach that may be used for the automated generation of flow cytometry data files containing information on single events about a virtually infinite number of parameters has already been done by Orfão et al. and opens the door for all applications of multiparameter flow cytometry for which a large number of parameters are needed, provided the fact that the cell population (or cell populations) of interest could be identified with a relatively limited number of markers. Thus, by comparing the expression of four cytokines (IL-2, IL-17, TNF- $\alpha$ and IFN- $\gamma$ ), we demonstrated that the major Th17 subpopulation in BM only produces IL-17, while in normal peripheral tissues, this subpopulation was significantly smaller, particularly in LN. On the other hand, in normal PB, there was an increase in the Th17 subpopulations producing TNF- $\alpha$ plus IL-2 and only producing TNF- $\alpha$, as well as a significant increase in the subset simultaneously producing all the four cytokines. In normal LN, this latter subpopulation was found even more represented, alongside the major Th17 subpopulation producing TNF- $\alpha$ plus IL-2 as compared to BM (Fig. 2).

Our findings suggest a functional heterogeneity and plasticity of Th17 cells, which seems to reflect the different environmental conditions found in the three normal tissues studied. In fact, Th17 cells retained in the BM appear to show a reduced ability to produce IL-17 (Table 2), and almost all do not express the type 1 cytokines studied, suggesting a protective environment with minimal contact with antigen and cytokine stimulation, and a strong fidelity lineage. In contrast, in peripheral tissues, Th17 cells can be dynamically induced to proliferate and become functional, activated according to various stimulus and interaction with antigen-APCs, resulting in a memory or effector phenotype characterized by an increased ability to co-produce cytokines, particularly in LN where "polyfunctional" Th17 cells could play an important homeostatic role. Thus, as also reported by others [24-26], our findings seem to support a notable flexibility between peripheral Th17 and Th1 cells or/and a shared early differentiation of Th1 and Th17 cells from naïve CD4+ T cell. In this sense, as Th1 subsets are thought to be the crucial player for most of the organspecific autoimmune diseases, these peripheral "Th17/Th1" subpopulations may be involved in the induction of autoimmunity and inflammatory reactions and represent a highly pathogenic effector $\mathrm{T}$ cell subsets. Thus, once the functional profile of normal Th17 cells is defined, we focus our work in their evaluation in a group of SLE patients with different activity diseases using the automated method described previously.

In inactive SLE, the Th17 cells producing only TNF- $\alpha$ were significantly lower than in normal controls. Compared to controls and active SLE, in inactive disease, there was an overall increase in the proportion of Th17 subpopulation co-producing TNF- $\alpha / \mathrm{IL}-2$ with a consequently decrease in the proportion of Th17 expressing TNF- $\alpha$ alone or IFN- $\gamma /$ IL-2 (Fig. 3). Interestingly, alterations in the frequency of pro-inflammatory cytokine-producing Th17 subpopulations, particularly the increased proportion of Th17 cells producing TNF- $\alpha / \mathrm{IL}-2$ observed in inactive SLE, might have important functional implications as these cytokines may exhibit a potent synergy with IL-17 for pro-inflammatory effects [27].

In active SLE patients, we found a similar subpopulation distribution to that observed in normal $\mathrm{PB}$, although showing a trend towards a higher proportion of Th17 cells producing IL-17 alone when compared to inactive SLE and normal controls, reflecting the decrease proportion of Th17 subpopulations expressing TNF- $\alpha /$ IL-2 and TNF- $\alpha$ (Fig. 3). Actually, in line with the notable cytokine profile plasticity presented by normal peripheral Th17 cells and taking into account the enhancement of Th2 cell function found previously by us in active SLE patients, [28] along with the miscellaneous immunosuppressive drugs received by those patients $[29,30]$, a suppressive effect may be exerted on Th17 polyfunctional effector phenotype, resulting in a decrease proportion of those subpopulations expressing TNF- $\alpha / \mathrm{IL}-2$ and TNF- $\alpha$. Especially, those findings may point to the strong fidelity lineage of Th17 in active disease SLE or even a terminal differentiation stage related with a repeated antigen challenge as compared to inactive SLE or normal control groups. Furthermore, consistent with other human studies [31, 32] that have 
demonstrated a lower number of Th1 cells in active SLE patients, our data suggest that the cytokine profile of Th17 cells may be essential for synergistically induced Th1-type cytokines and also potentially be distinct according to the pro-inflammatory environment. Thus, the local cytokine milieu may induce new transcription factors and modify cytokine production even in fully polarized effector T-cell lineages. The data recorded here provide evidences to show that, when exposed to certain cytokine milieus, Th17 cells may acquire the capacity to express not only IL-17 but also different type 1 cytokines, such as IL-2, TNF- $\alpha$ and IFN- $\gamma$, thus favouring the shifting of these cells toward Th1 phenotype, which is particularly visible in those patients with inactive disease. In this regard, preliminary data suggest that these "Th17/Th1" cells appear to be differently influenced by environmental factors to exhibit dual Th1 and Th17 functions and to be related with disease activity in SLE patients, denoting their potential application in clinical trials as a therapeutic target.

In conclusion, it is conceivable that Th17 cells are responsive to local cytokine milieus and sufficiently flexible to acquire the ability to produce type 1 cytokine, contributing in a pathologic condition to acute and chronic inflammation mainly in the context of Th1-initiated disease, herein creating a mixed "Th1/Th17" pathology and reduced dominance of Th1 in active SLE. Therefore, the data presented provide a detailed functional characterization of human Th17 isolated from PB of SLE patients, as well as from various normal tissues, using a new statistical approach for the automated generation of flow cytometry data files that clearly show its great utility in future studies that would complete the plasticity character of Th17 cells. In line with this, in the future, it would be important to determine in a large study, including also non-treated patients, if these eight $\mathrm{T}$ cell subsets, so-called Th1/Th17 cells, can be considered as distinct and stable lineage of $\mathrm{CD}^{+} \mathrm{T}$ effector cells that are committed to a certain lineage or whether they are an intermediate activation state destined to become true Th1 or Th17 cells and what might be their functional relevance in the clinical heterogeneity of SLE patients, namely, concerning organ involvement and monitoring patient treatment response.

\section{Disclosures None.}

\section{References}

1. Harrington LE, Mangan PR, Weaver CT (2006) Expanding the effector CD4 T-cell repertoire: the Th17 lineage. Curr Opin Immunol 18:349-356

2. Harrington LE, Hatton RD, Mangan PR et al (2005) Interleukin 17-producing CD4+ effector T cells develop via a lineage distinct from the T helper type 1 and 2 lineages. Nat Immunol 6:11231132

3. Park H, Li Z, Yang XO et al (2005) A distinct lineage of CD4 T cells regulates tissue inflammation by producing interleukin 17 . Nat Immunol 11:1133-1141

4. Shi G, Cox CA, Vistica BP, Tan C, Wawrousek EF, Gery I (2008) Phenotype switching by inflammation-inducing polarized Th17 cells, but not by Th1 cells. J Immunol 181:7205-7213

5. Lee YK, Turner H, Maynard CL et al (2009) Late developmental plasticity in the $\mathrm{T}$ helper 17 lineage. Immunity 30:92-107

6. Lexberg MH, Taubner A, Albrecht I et al (2010) IFN- $\gamma$ and IL-12 synergize to convert in vivo generated Th17 into Th1/Th17 cells. Eur J Immunol 40:3017-3027

7. Yang XO et al (2008) Molecular antagonism and plasticity of regulatory and inflammatory T cell programs. Immunity 29:44-56

8. Walline CC, Kanakasabai S, Bright JJ (2011) IL-7R $\alpha$ confers susceptibility to experimental autoimmune encephalomyelitis. Genes Immun 12:1-14

9. Miossec P (2003) Interleukin-17 in rheumatoid arthritis: if T cells were to contribute to inflammation and destruction through synergy. Arthritis Rheum 48:594-601

10. RL Van bezooijen, Farih-Sips HC, Papapoulos SE, Lowik CW (1999) Interleukin-17: a new bone acting cytokine in vitro. J Bone Miner Res 14:1513-1521

11. Shih DQ, Targan SR, McGovern D (2008) Recent advances in IBD pathogenesis: genetics and immunobiology. Curr Gastroenterol Rep $10: 568-575$

12. Kurasawa K, Hirose K, Sano H et al (2000) Increased interleukin17 production in patients with systemic sclerosis. Arthritis Rheum 43:2455-2463

13. Crispin JC, Oukka M, Bayliss G et al (2008) Expanded double negative $\mathrm{T}$ cells in patients with systemic lupus erythematosus produce IL-17 and infiltrate the kidneys. J Immunol 181:8761-8766

14. Wong CK, Lit LC, Tam LS, Li EK, Wong PT, Lam CW (2008) Hyperproduction of IL-23 and IL-17 in patients with systemic lupus erythematosus: implications for Th17-mediated inflammation in auto-immunity. Clin Immunol 127:385-393

15. Dardalhon V, Korn T, Kuchroo VK, Anderson AC (2008) Role of Th1 and Th17 cells in organ-specific autoimmunity. J Autoimmun 31:252-256

16. Annunziato F, Cosmi L, Santarlasci V et al (2007) Phenotypic and functional features of human Th17 cells. J Exp Med 204:18491861

17. Pedreira CE, Costa ES, Barrena S et al (2008) Generation of flow cytometry data files with a potentially infinite number of dimensions. EuroFlow Consortium. Cytometry A 73:834-846

18. Hochberg MC (1997) Updating the American College of Rheumatology revised criteria for the classification of systemic lupus erythematosus. Arthritis Rheum 40:1725

19. Bombardier C, Gladman DD, Urowitz MB, Caron D, Chang CH (1992) Derivation of the SLEDAI. A disease activity index for lupus patients. The Committee on Prognosis Studies in SLE. Arthritis Rheum 35:630-640

20. Gladman DD, Ibanez D, Urowitz MB (2002) Systemic lupus erythematosus disease activity index 2000. J Rheumatol 29:288291

21. Griffiths B, Mosca M, Gordon C (2005) Assessment of patients with systemic lupus erythematosus and the use of lupus disease activity indices. Best Pract Res Clin Rheumatol 19:685-708

22. Yang Y, Weiner J, Liu Y et al (2009) T-bet is essential for encephalitogenicity of both Th1 and Th17 cells. J Exp Med 206:1549-1564

23. Evans HG, Suddason T, Jackson I, Taams LS, Lord GM (2007) Optimal induction of $\mathrm{T}$ helper 17 cells in humans requires $\mathrm{T}$ cell receptor ligation in the context of toll-like receptor-activated monocytes. Proc Natl Acad Sci U S A 104:17034-17039 
24. Garrett-Sinha LA, John S, Gaffen SL (2008) IL-17 and the Th17 lineage in systemic lupus erythematosus. Curr Opin Rheumatol 20:519-525

25. Mackenzie BS, Kastelein RA, Cua DJ (2006) Understanding the IL-23-IL-17 immune pathway. Trends Immunol 27:17-23

26. Zhou L, Chong MM, Littman DR (2009) Plasticity of CD4+ T cell lineage differentiation. Immunity 30:646-655

27. Shen F, Gaffen SL (2008) Structure-function relationships in the IL-17 receptor: implications for signal transduction and therapy. Cytokine 41:92-104

28. Henriques A, Inês L, Couto M et al (2010) Frequency and functional activity of Th17, Tc17 and other T cell subsets in systemic lupus erythematosus. Cell Immunol 264:97-103
29. Ermann J, Bermas BL (2007) The biology behind the new therapies for SLE. Int J Clin Pract 61:2113-2119

30. Ramirez F, Fowell DJ, Puklavec M, Simmonds S, Mason D (1996) Glucocorticoids promote a TH2 cytokine response by CD4+ T cells in vitro. J Immunol 156:2406-2412

31. Akahoshi M, Nakashima H, Tanaka Y et al (1999) Th1/Th2 balance of peripheral $\mathrm{T}$ helper cells in systemic lupus erythematosus. Arthritis Rheum 42:1644-1648

32. Chen S, Hu D, Shi X, Shen N, Gu Y, Bao C (2000) The relationship between Th1/Th2-type cells and disease activity in patients with systemic lupus erythematosus. Chin Med J 113:877880 